\title{
Numerical Verification of Transition's Energies of Excitons in Quantum Well of ZnO with the Finite Difference Method
}

\author{
Bassirou Lô, Serigne Bira Gueye \\ Department of Physics, Sciences and Technics Faculty, University Cheikh Anta Diop UCAD, Dakar, Senegal \\ Email: lobass57@gmail.com, sbirag@gmail.com
}

Received 22 October 2015; accepted 23 February 2016; published 26 February 2016

Copyright (C) 2016 by authors and Scientific Research Publishing Inc.

This work is licensed under the Creative Commons Attribution International License (CC BY).

http://creativecommons.org/licenses/by/4.0/

(c) (i) Open Access

\begin{abstract}
This paper shows that the experimental results of quantum well energy transitions can be found numerically. The cases of several $\mathrm{ZnO}-\mathrm{ZnMgO}$ wells are considered and their excitonic transition energies were calculated using the finite difference method. In that way, the one-dimensional Schrödinger equation has been solved by using the BLAS and LAPACK libraries. The numerical results are in good agreement with the experimental ones.
\end{abstract}

\section{Keywords}

Quantum Well Zn0-ZnMg0, Finite Difference Method, Exciton, Transition Energy

\section{Introduction}

$\mathrm{ZnO}$ is a very abundant material in nature, with very interesting physiochemical properties: it is non-toxic and presents high chemical stability. It possesses a direct and large band gap (3.37 eV) at room temperature. Its exciton binding energy of the order of $60 \mathrm{meV}$, enables the design of laser devices operating at room temperature. Its heterostructures are very interesting for optoelectronic applications.

In general, the search for the eigenstates of the Hamiltonian is complex. Even analytically soluble case of the hydrogen atom is not strictly in simple form if we neglect the coupling with the electromagnetic field. The Schrödinger equation, even in one dimension, admits precious few analytic solutions so that in the other cases, it is necessary to use various approximation techniques. Perturbation theory provides analytical expressions in the form of asymptotic expansions around undisturbed exactly solvable problem. Numerical analysis allows the exploration of inaccessible situations by perturbation theory. In fact the continuous Schrödinger equation is not always the most reasonable choice for realize modeling of semiconductors quantum well, superlatice and nanostructures devices. However, the dependency of the energy state on the wave vector dispersion equation for a 
bulk semiconductor is very close to a discrete model. And as we know, the realistic physics of the Schrödinger equation arises from its equivalence to a tightbiding model for crystalline solid [1], in both the bulk and quantum confinement cases. Furthermore, day-to-day, the analytic solution for tight-binding model of quantum wells has not been presented.

This study presents a numerical model that allows retrieving transition energies of excitons measured in a $\mathrm{ZnO}-\mathrm{ZnMgO}$ or other quantum-well. This model is based on the finite difference method [2] [3].

First, we will present the results of experimental measurements of transition energies of excitons in $\mathrm{ZnO}$ quantum well. Then, we propose an one-dimensional model of potential barrier which describes the electronic behavior of these wells. Thus, the corresponding model, which is governed by the Schrödinger equation in steady state, is solved here with the finite difference method. Finally, we compare the numerical results with the experimental results.

\section{Experimental Results and Model}

ZnO-ZnMgO quantum wells have been realized with very high crystallographic and optical quality.

\subsection{Experimental Details: Growth and Structural Properties}

The samples were grown by plasma-assisted MBE, the metals ( $\mathrm{Zn}$ and $\mathrm{Mg}$ ) being evaporated using Knudsen cells and atomic oxygen (O) being activated in a radio-frequency plasma cell [4]. The residual carrier concentration in the vicinity of the ZnO QWs is estimated to be less than $10^{16} \mathrm{~cm}^{3}$.

We considered three samples. The first contains only one quantum well of width $L_{w}=1.6 \mathrm{~nm}$. The second sample contains two quantum wells of width $2.1 \mathrm{~nm}$ and $3.6 \mathrm{~nm}$, respectively. The last and third sample possesses one quantum well of width $L_{w}=10 \mathrm{~nm}$. A schematic diagram (Figure 1) and details (Table 1) of the quantum structure are presented.

The Mg content of the barrier layers was in the range of $21 \%-22 \%$, varying slightly from sample to sample. The QWs were grown at $480^{\circ} \mathrm{C}$ i.e. the investigated samples do not contain any cubic inclusions.

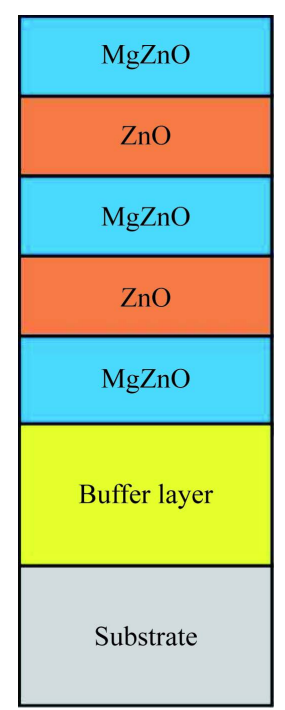

Figure 1. Quantum structure.

Table 1. Details of quantum structure.

\begin{tabular}{ccccc}
\hline QW width (nm) & 1.6 & 2.1 & 3.6 & 10 \\
\hline Mg content (\%) & 20 & 25 & 25 & 17 \\
Barrier thickness (nm) & 200 & 95 & 95 & 130 \\
Buffer thickness (nm) & 100 & 680 & 680 & 510 \\
\hline
\end{tabular}




\subsection{Structural Characterization and Optical Properties}

$\mathrm{X}$-ray reciprocal space maps of samples grown at $480^{\circ} \mathrm{C}$ were performed on the $\overline{1} 0.5$ and $\overline{1} 1.4$ reflections. While these maps suggest that the ZnMgO barriers are at least partially relaxed for $\mathrm{Mg}$ contents larger than $40 \%$, heterostructures having $\mathrm{Mg}$ contents in the range of $20 \%$ are found to be perfectly lattice matched [4]. The highquality ZnO-ZnMgO QWs grown on sapphire substrate $\left(\mathrm{Al}_{2} \mathrm{O}_{3}(0001)\right)$, was demonstrated by means as RHEED, AFM and PL studies. PL spectra of one of these samples recorded at $10 \mathrm{~K}$ are displayed in Figure 2. The high energy peak (3.82 eV) corresponds to the PL emission of the barrier layer with Mg content of $21 \%$ - 22\%, while the emission at $3.37 \mathrm{eV}$ corresponds to the free exciton recombination from the $\mathrm{ZnO}$ template and the other line $3.535 \mathrm{eV}$ is the excitonic emission of the QW of width $1.6 \mathrm{~nm}$.

Figure 3 concerns the low temperature PL spectra from a $10 \mathrm{~nm}$ thick QW (b) embedded in a $\mathrm{Zn}_{0.83} \mathrm{Mg}_{0.17} \mathrm{O}$

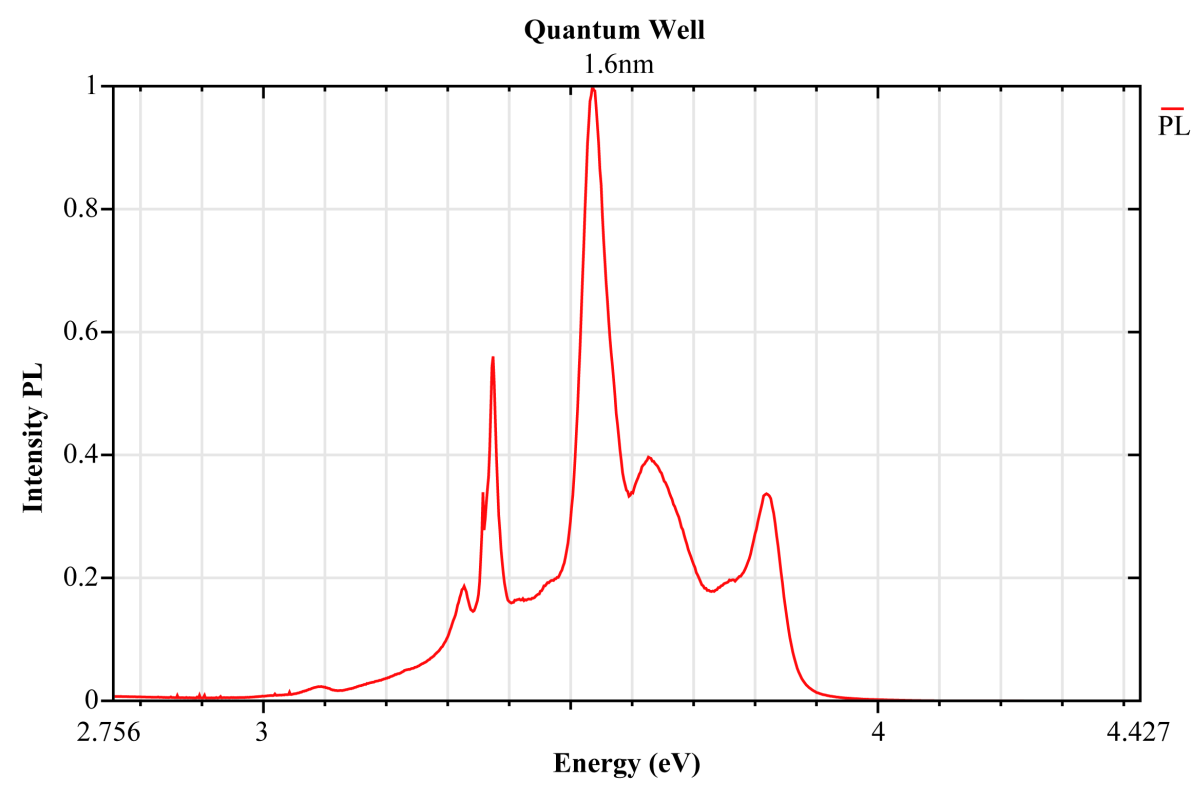

Figure 2. PL spectrum of $\mathrm{ZnO}$ QW $\left(L_{w}=1.6 \mathrm{~nm}\right)$.

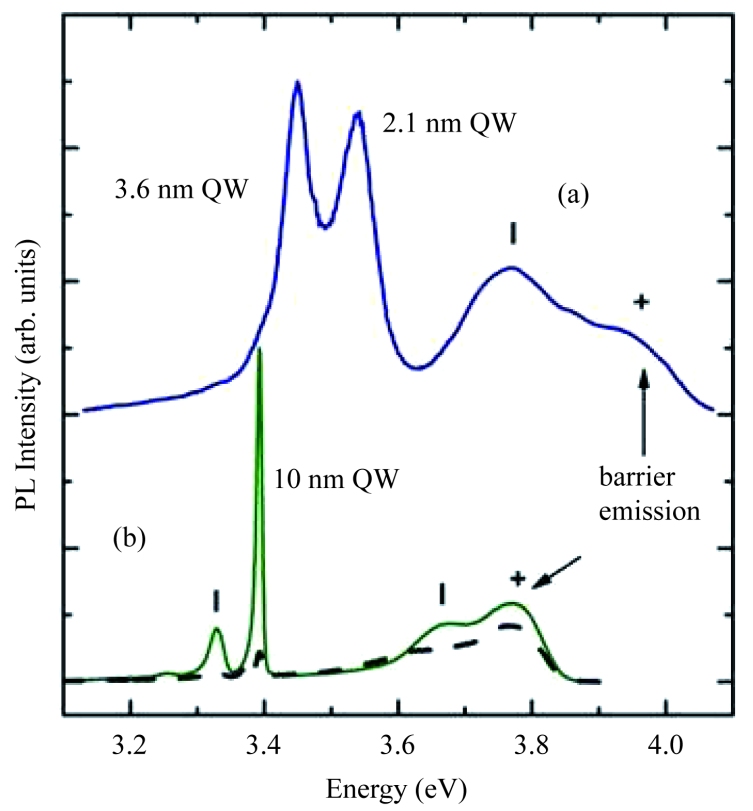

Figure 3. QWs $3.6 \mathrm{~nm}, 2.1 \mathrm{~nm}$, and $10 \mathrm{~nm}$. 
barrier layer grown on R-plane sapphire substrate and from a sample containing two QWs (a) [5]. The excitonic emission from the $10 \mathrm{~nm}$ QW emits at $3.39 \mathrm{eV}$, which is also the energy of the excitonic band gap of A-plane $\mathrm{ZnO}$, while the $\mathrm{PL}$ of the $\mathrm{Zn}_{0.83} \mathrm{Mg}_{0.17} \mathrm{O}$ barrier layer peaks at $3.82 \mathrm{eV}$.

Figure 3(a) corresponds to the PL lines of a sample containing two QWs of width (3.6 nm) and $(2.1 \mathrm{~nm})$, emitting at $3.44 \mathrm{eV}$ and $3.451 \mathrm{eV}$, respectively. The widths of the QWs were chosen to be sufficiently different so that the corresponding PL emission lines are well separated [5]. So, for the band conduction electrons, a potential barrier of $430 \mathrm{meV}$ and $447 \mathrm{meV}$ is formed. The behavior of the electron in the QW is described by the Schrödinger equation:

$$
\left[-\frac{\mathrm{d}}{\mathrm{d} x}\left(\frac{\hbar^{2}}{2 m^{*}(x)} \frac{\mathrm{d}}{\mathrm{d} x}\right)+V(x)\right] \psi_{n}(x)=E_{n} \psi_{n}(x)
$$

where $E_{n}$ is the electron confinement energy. The correction potential energy due to the difference effective mass between the barrier and the quantum well is neglected. The model we propose able to find the energy confinement of the electron in the QW and then their stationary states. Contrary to other computational approaches [6], it is more direct. A schematic description of the potential is presented in Figure 4.

\section{Use of the Finite Difference Method}

The Finite Difference method for solving the 1D Time Independent Schrödinger Equation is presented. This method is a simple and very important tool for physics and engineering where the Schrödinger equation appears very often in the description of certain phenomena [7].

\subsection{Stationary States in 1D Potential}

This is described by the following equation:

$$
-\frac{\hbar^{2}}{2 m_{e}} \frac{\partial^{2}}{\partial x^{2}} \psi_{n}(x)+\left[V(x)-E_{n}\right] \psi_{n}(x)=0 .
$$

This is equivalent to

$$
\frac{\partial^{2}}{\partial x^{2}} \psi_{n}(x)-\left[\frac{2 m_{e}}{\hbar^{2}} V(x)-\frac{2 m_{e}}{\hbar^{2}} E_{n}\right] \psi_{n}(x)=0 .
$$

This latter can be normalized with

$$
\alpha=\frac{2 m_{e}}{\hbar^{2}}, \tilde{V}(x)=-\alpha V(x) \text { and } \tilde{E}_{n}=\alpha E_{n} .
$$

Thus we get

$$
\frac{\partial^{2}}{\partial x^{2}} \psi_{n}(x)+\left[\tilde{V}(x)+\tilde{E}_{n}\right] \psi_{n}(x)=0
$$

this equation will be solved with the Finite Difference Method (FDM).

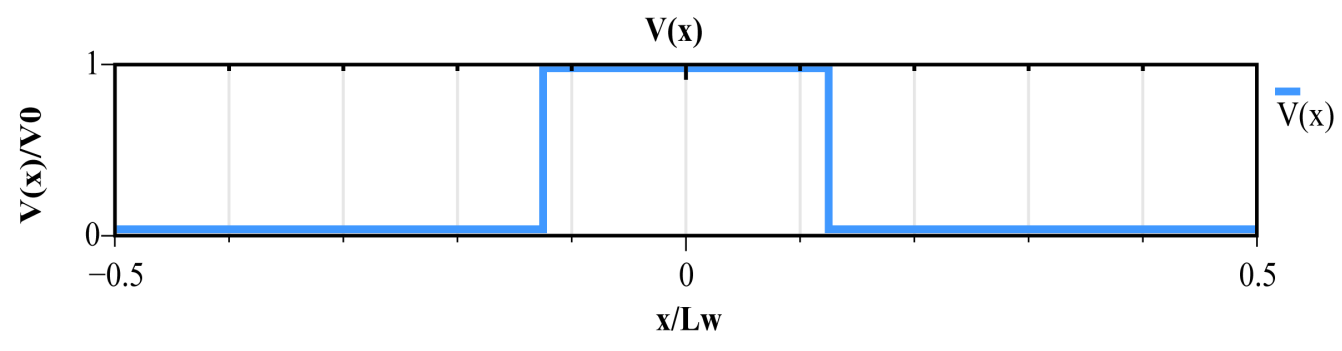

Figure 4. Potential barrier. 


\subsection{Finite Difference Method}

We consider a function specified $\psi_{n}(x)$ which satisfies the Schrödinger equation, in the interval ]$-L_{w} / 2, L_{w} / 2\left[\cdot \psi_{n}(x)\right.$ fulfills the boundary conditions $\psi_{n}\left(-L_{w} / 2\right)=\psi_{n}^{0}$ and $\psi_{n}\left(L_{w} / 2\right)=\psi_{n}^{N+1}$. We consider an one-dimensional mesh with $N+2$ points $x_{i}=-L_{w} / 2+i * \Delta x$, where $\Delta x=\frac{L_{w}}{N+1}$ and we define $\psi_{n}^{i} \approx \psi_{n}\left(x_{i}\right), \quad \tilde{V}^{i}=f\left(x_{i}\right) ; i=0,1, \cdots, N+1$.

The FDM is based on the Taylor expansion. So, with the centered difference approximation, the second order derivative of the stationary wave functions can be approximated by the following:

$$
\frac{\partial^{2} \psi_{n}(x)}{\partial x^{2}}=\frac{\psi_{n}(x+\Delta x)-2 \psi_{n}(x)+\psi_{n}(x-\Delta x)}{\Delta x^{2}}+O\left(\Delta x^{2}\right) .
$$

Thus, the 1D Time Independent Schrödinger Equation becomes a set of algebraic equations

$$
\frac{\psi_{n}^{i+1}-2 \psi_{n}^{i}+\psi_{n}^{i-1}}{\Delta x^{2}}+\left[\tilde{V}^{i}+\tilde{E}_{n}\right] \psi_{n}^{i}=0, \quad i=1,2, \cdots, N .
$$

This is equivalent to following

$$
\left.\psi_{n}^{i+1}+\left(-2+\Delta x^{2} \tilde{V}^{i}\right)\right) \psi_{n}^{i}+\psi_{n}^{i-1}=-\Delta x^{2} \tilde{E}_{n} \psi_{n}^{i}, \quad i=1,2, \cdots, N .
$$

Defining

$$
d_{i}=\left(2-\Delta x^{2} \tilde{V}^{i}\right) \quad i=1,2, \cdots, N
$$

one gets a linear system of $N$ equations, which can be written in a matrix form [3].

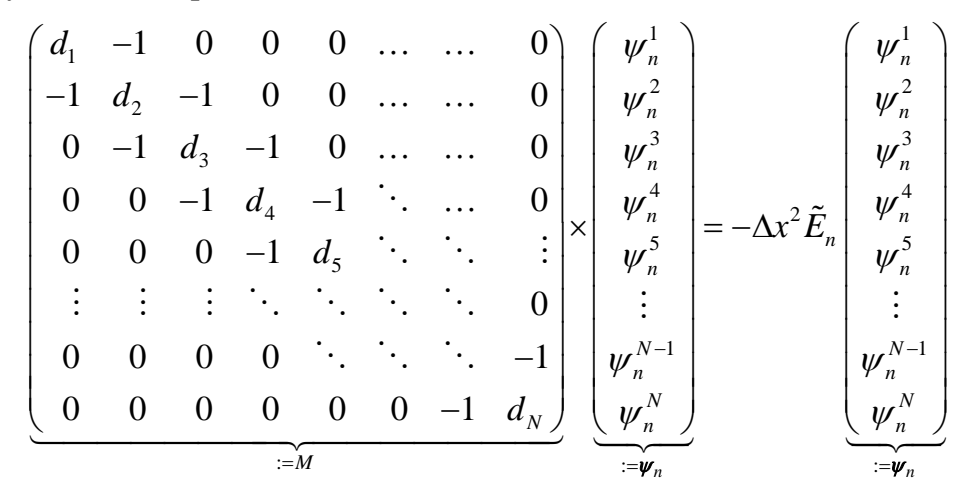

To solve this $1 \mathrm{D}$ equation means to determine the eigenvalues $E_{n}$ and thus the eigenvectors $\boldsymbol{\psi}_{n}$. Equation (10) has been solved with FORTRAN, using the librairies of BLAS and LAPACK for eigenvalue/eigenvactor problems. Particularly, the parameters of our QWs allow us to determine the diagonal elements of the symmetric matrix $M$. Thus, the eigenvalues of $M$ have been calculated and the energy levels $E_{n}$ have been determined. The obtained results are shown in Table 2.

\section{Verification and Discussions}

A verification of the proposed method is done, considering the results of three samples. It concerns four quantum wells whose widths are $1.6 \mathrm{~nm}, 2.1 \mathrm{~nm}, 3.6 \mathrm{~nm}$ et $10 \mathrm{~nm}$; respectively.

The numerical calculation, carried out for all the wells, allowed to recover the experimental results. If we consider the quantum well of width $1.6 \mathrm{~nm}$, the expected emission energies are between 1.45 and $1.65 \mathrm{eV}$, according to the experimental measurements. The energy levels E1 and E2, calculated with the method of finite difference, correspond to emission energies of $373 \mathrm{meV}$ and $168 \mathrm{meV}$, respectively. These values obtained are consistent with the experimental results. For the other quantum wells, this agreement between numerical result and experience can be observed. These results are summarized in Table 2.

The proposed numerical method using the finite difference method allows retrieving the experimental values of emission energies of unstrained $\mathrm{ZnO}-\mathrm{ZnMgO}$ quantum wells where the Stark effect has been neglected. 
Table 2. Numerical results.

\begin{tabular}{|c|c|c|c|c|}
\hline Energy [mev]\QW width & $\begin{array}{c}\mathrm{L}_{\mathrm{w}}=1.6 \mathrm{~nm} \\
\mathrm{~V}_{0}=447 \mathrm{mev}\end{array}$ & $\begin{array}{c}\mathrm{L}_{\mathrm{w}}=2.1 \mathrm{~nm} \\
\mathrm{~V}_{0}=430 \mathrm{mev}\end{array}$ & $\begin{array}{c}\mathrm{L}_{\mathrm{w}}=3.6 \mathrm{~nm} \\
\mathrm{~V}_{0}=430 \mathrm{mev}\end{array}$ & $\begin{array}{c}\mathrm{L}_{\mathrm{w}}=10 \mathrm{~nm} \\
\mathrm{~V}_{0}=430 \mathrm{mev}\end{array}$ \\
\hline E1 & -373 & -381 & -410 & -427 \\
\hline E2 & -168 & -241 & -350 & -417 \\
\hline E3 & & -42 & -253 & -402 \\
\hline E4 & & & -126 & -381 \\
\hline E5 & & & & -354 \\
\hline E6 & & & & -323 \\
\hline E7 & & & & -286 \\
\hline E8 & & & & -254 \\
\hline E9 & & & & -200 \\
\hline E10 & & & & -152 \\
\hline E11 & & & & -102 \\
\hline E12 & & & & -50 \\
\hline E13 & & & & -1 \\
\hline
\end{tabular}

\section{Conclusion}

In summary, we have shown in this paper that the model of one-dimensional quantum wells allows finding excitonic energy levels determined experimentally. By solving the Schrödinger equation with the finite difference method, we could recover experimental energy levels with high accuracy. A subsequent study could be interested in the case of optically coupled quantum wells, superlatices and atomic latices with sinusoidal potential in plane. This work can be extended to the diffraction of Gaussian wave under Fraunhofer condition.

\section{References}

[1] Boykin, T.B. (2001) American Journal of Physics, 69, 793-798. http://dx.doi.org/10.1119/1.1344169

[2] Boykin, T.B. and Klimeck, G. (2004) The Discretized Schrodinger Equation and Simple Models for Semiconductor Quantum Wells.

[3] LeVeque, R.J. (2007) Finite Difference Method for Ordinary and Partial Differential Equations, Steady State and Time Dependent Problems. SIAM, 15-16.

[4] Morhain, C., Tang, X., Teisseire-Doninelli, M., Lo, B., Laügt, M., Chauveau, J.-M., Vinter, B., Tottereau, O., Vennéguès, P., Deparis, C. and Neu, G. (2005) Supperlattices and Microstructures, 38, 455-463. http://dx.doi.org/10.1016/j.spmi.2005.08.055

[5] Chauveau, J.-M., Laügt, M., Venneguès, P., Teisseire, M., Lo, B., Deparis, C., Morhain, C. and Vinter, B. (2008) Semiconductor Science and Technology, 23, Article ID: 035005. http://dx.doi.org/10.1088/0268-1242/23/3/035005

[6] Gruber, T., Kirchner, C., Kling, R., Reuss, F. and Waag, A. (2004) Applied Physics Letters, 84, 5359. http://dx.doi.org/10.1063/1.1767273

[7] Erwin, S. (1926) Physical Review, 28, 1049. http://dx.doi.org/10.1103/PhysRev.28.1049 